\title{
Using principal component analysis to reduce complex datasets produced by robotic technology in healthy participants
}

\author{
Michael D. Wood', Leif E. R. Simmatis ${ }^{1}$, J. Gordon Boyd ${ }^{1,2,3}$, Stephen H. Scott ${ }^{1}$ and Jill A. Jacobson ${ }^{4 *}$ (D)
}

\begin{abstract}
Background: The KINARM robot produces a granular dataset of participant performance metrics associated with proprioceptive, motor, visuospatial, and executive function. This comprehensive battery includes several behavioral tasks that each generate 9 to 20 metrics of performance. Therefore, the entire battery of tasks generates well over 100 metrics per participant, which can make clinical interpretation challenging. Therefore, we sought to reduce these multivariate data by applying principal component analysis (PCA) to increase interpretability while minimizing information loss.

Methods: Healthy right-hand dominant participants were assessed using a bilateral KINARM end-point robot. Subjects ( $N s=101-208$ ) were assessed using 6 behavioral tasks and automated software generated 9 to 20 metrics related to the spatial and temporal aspects of subject performance. Data from these metrics were converted to Z-scores prior to PCA. The number of components was determined from scree plots and parallel analysis, with interpretability considered as a qualitative criterion. Rotation type (orthogonal vs oblique) was decided on a per task basis.
\end{abstract}

Results: The KINARM performance data, per task, was substantially reduced (range 67-79\%), while still accounting for a large amount of variance (range 70-82\%). The number of KINARM parameters reduced to 3 components for 5 out of 6 tasks and to 5 components for the sixth task. Many components were comprised of KINARM parameters with high loadings and only some cross loadings were observed, which demonstrates a strong separation of components.

Conclusions: Complex participant data produced by the KINARM robot can be reduced into a small number of interpretable components by using PCA. Future applications of PCA may offer potential insight into specific patterns of sensorimotor impairment among patient populations.

Keywords: KINARM, End-point robot, Principal component analysis, Data reduction

\section{Background}

Robotic technology, such as KINARM (BKIN Technologies, Kingston, ON, Canada), provides objective metrics using the participants' upper limbs to assess proprioception and sensorimotor function, as well as executive function. In stroke survivors, this technology has identified subtle neurocognitive deficits not apparent on routine clinical testing [1], and various KINARM tasks have

\footnotetext{
* Correspondence: jill.jacobson@queensu.ca

${ }^{4}$ Department of Psychology, Queen's University, 318 Craine Hall, 62 Arch Street, Kingston, ON K7L 3N6, Canada

Full list of author information is available at the end of the article
}

been administered to multiple patient populations (e.g., traumatic brain injury, fetal alcohol spectrum disorder) [2-5]. The KINARM behavioral battery (KINARM Standard Tests $\left.{ }^{\mathrm{s}}\right)$ currently consists of 9 tasks that include automated data analysis routines. Within each task, up to 20 performance items (e.g., reaction time) are calculated, leading to the potential generation of over 100 metrics per participant. However, when assessing performance deficits that are indicative of adverse neurological outcomes among participants, this granular and complex performance output may impede deriving meaningful interpretations.

(c) The Author(s). 2018 Open Access This article is distributed under the terms of the Creative Commons Attribution 4.0 International License (http://creativecommons.org/licenses/by/4.0/), which permits unrestricted use, distribution, and 
Principal component analysis (PCA) is a data reduction technique used to identify linear combinations of measured variables that account for the most overall variance in responses [6-8]. The first principal component accounts for the largest amount of variance, followed by the second, and so forth [9]. PCA is best used when the measured variables are theorized to be causal or formative indicators of the overarching construct rather than reflective or effects of it, which would be better assessed using factor analysis (FA). Because performance on the tasks determine a participant's level of functioning rather than the other way around, PCA is the more appropriate technique for the KINARM battery than is FA.

The primary objective of this study was to reduce the dimensionality of healthy participant data produced by the KINARM End-Point robot by using PCA. This analysis should increase overall interpretability by reducing redundant KINARM parameters into behaviorally meaningful components, which has the potential to demonstrate the clinical utility of PCA by capturing a minimal number of performance measures that could assist with the characterization of deficits among various patient populations.

\section{Methods}

\section{Participant recruitment}

Healthy participants were community-based and were randomly recruited via advertisements on lab and departmental websites, as well as in local classifieds (online and print). Trained research staff screened each adult participant ( $>17$ years old) to ensure that task instructions could be easily understood, no prior neurological deficits were reported, and that subjects had no prior medical conditions that could affect upper limb mobility (see Additional file 1). Once enrolled, participants were then assessed by our research staff at one of two sites: Kingston Health Sciences Centre or Queen's University, both sites in Kingston, ON, Canada. See Table 2 for detailed demographics (e.g., age, gender, education). The Queen's University and Affiliated Hospitals Health Sciences Research Ethics Board approved recruitment and assessment of these participants. Informed consent was obtained from each participant prior to the KINARM assessment.

\section{Robotic assessment}

Participants were seated, in a height-adjustable chair that was locked in place, at a two-dimensional virtual reality system that displayed each task in the horizontal plane. As shown in Fig. 1, participants' vision of their hands and arms was occluded, their head was positioned in the center of the visual field, and visual feedback of hand position (when provided) was represented on the screen by a white circle. Participants were instructed to grasp onto the KINARM End-Point robotic handles (BKIN Technologies Ltd., Kingston, ON, Canada), permitting free movement in the horizontal plane without anti-gravity support at proximal or distal arm segments. A trained operator described each task, using a standardized script, before it was performed by the participant. Automated data collection and analysis software (Dexterit Version 3.6.2) measured and quantified subject performance. For each performance metric, the software computed a Z-score that accounted for age, sex, and handedness. Only performance metrics that could be normalized, and therefore converted to $\mathrm{Z}$ scores, were considered in the current analysis. We examined 6 tasks from the KINARM Standard Tests ${ }^{\mathrm{TM}}$, four tasks assessed upper-limb motor function in right-handed participants: visually guided reaching (VGR), object hit $(\mathrm{OH})$, object hit and avoid (OHA), and level 1 of ball on bar (BonB). The Arm position matching (APM) task assessed upper limb proprioception, and reverse visually guided reaching (RVGR) assessed cognitive-motor function. For detailed descriptions of all tasks, see Table 1. Within each task, approximately 9-20 performance metrics were produced, participants were instructed to take breaks as needed, and the assessment took $<1 \mathrm{~h}$ to complete. For detailed descriptions on all task parameters, see Additional file 2 or obtain from the BKIN Technologies Ltd. KINARM manual [10]. All included participants were selected based on their ability and understanding to follow task commands. Each task was comprised of various healthy participants, as not all participants completed each task. To reduce handedness as a potential confounding variable, only right hand dominant subjects were included in the analysis.

\section{Principal component analysis}

PCA analysis was conducted in $\mathrm{R}$ version 3.3.2 [11] using the psych package version 1.7.8 [12]. KINARM performance items were converted to Z-scores prior to PCA. For correlation matrices associated with each task, see Additional file 3. Scree plots and parallel analysis were examined to determine the number of components per KINARM task. Briefly, eigenvalues (variance explained by a component) were plotted in descending order to generate a scree plot $[13,14]$ per KINARM task. Parallel analysis was used to generate a random data set that possessed the same number of items and sample size as each KINARM task. Eigenvalues then were calculated for the random data, which also are graphed on the scree plot mentioned previously. The number of components prior to where the random data crosses the observed KINARM participant data are retained (i.e., retain the number of components that have eigenvalues larger than those from the simulated dataset) [13-16]. In PCA, if more than one component is retained, then multiple solutions for the parameters 

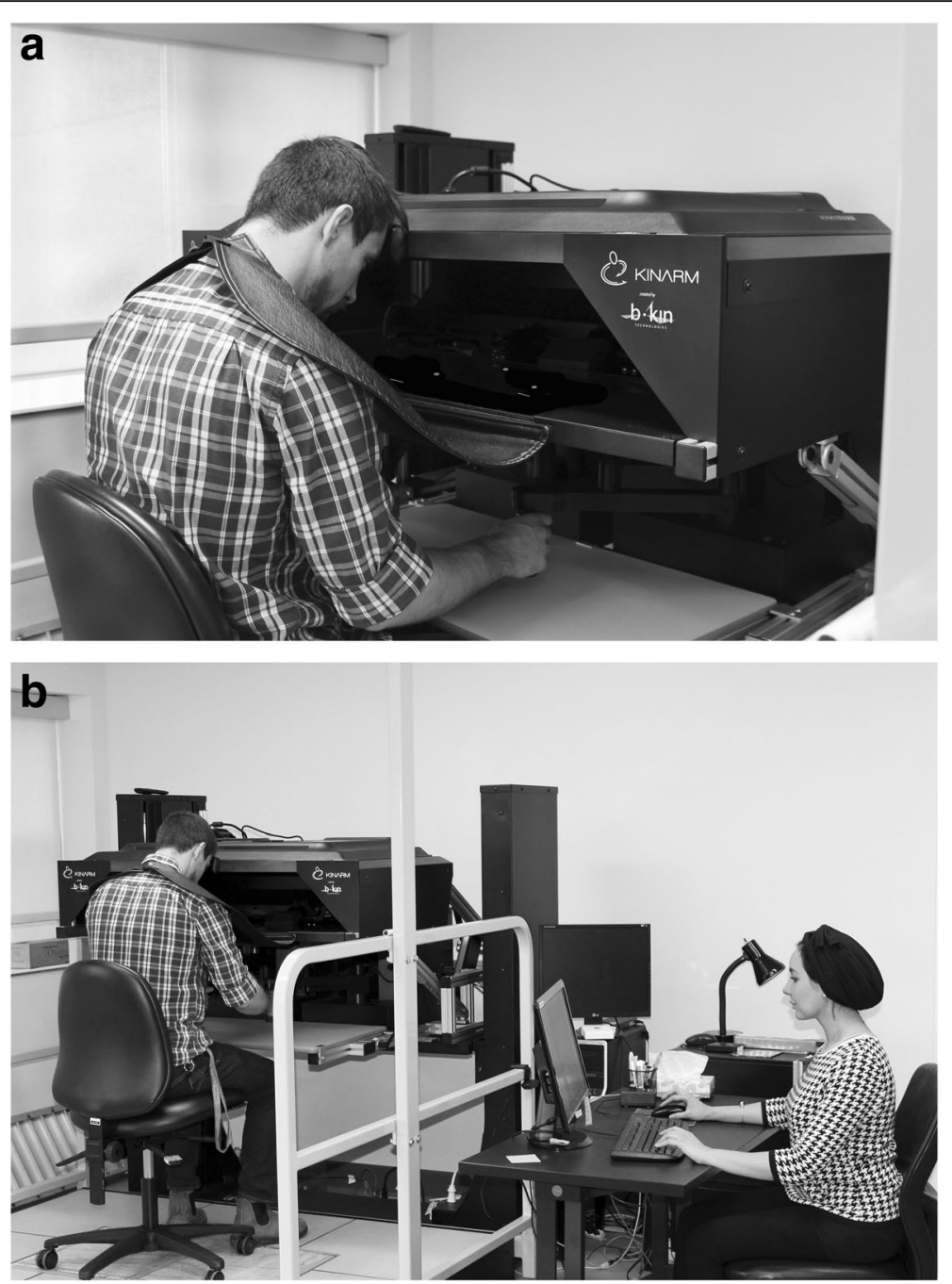

Fig. 1 KINARM End-Point robot set-up during participant assessment

would fit the data equally well. Rotation is used to help select the best solution. An oblique rotation (oblimin) [17], which allows for components to be correlated, first was conducted for each solution. If all intercomponent correlations were low $(<|0.30|)$, then an orthogonal rotation (varimax) [18] was used instead. A component loading was considered substantial if the loading was $\geq 0.40$. Only participants who were right hand dominant were chosen for this analysis, and only data from their dominant hand were used for PCA. To reduce possible practice effects for those participants who completed multiple assessments, we used the participant performance metrics from the first KINARM assessment only (i.e., for participants who completed the same task multiple times [repeated testing], we used their performance score only from the first assessment of that task). These identified components were then analyzed for interpretability, which was defined as parameters having substantial loadings on each component and groupings of performance parameters having behaviorally meaningful conclusions (e.g., the constructs of "total movement" and "posture/reaction time" being separable). To further characterize participant performance, components and their respective parameters that loaded highly were used to broadly generate intuitive labels for each component per task rather than successively numbering components.

\section{Results}

\section{Participant characteristics}

The number of participants across each task varied (Ns =101-208). Participant level of education consistently ranged from high school education to postdoctoral researcher. The percent of participants that were male ranged from 40 to $46 \%$, and the mean age of subjects (range: 41-46 years) was fairly consistent across all 
Table 1 KINARM Task Descriptions and Domains of Assessment

\begin{tabular}{|c|c|c|}
\hline Domain & Task & Description \\
\hline \multirow{4}{*}{ Motor } & $\begin{array}{l}\text { Visually guided reaching } \\
\text { (VGR) } \\
0\end{array}$ & $\begin{array}{l}\text { Participants grasp and move the right arm of the robot } \\
\text { (visual feedback is represented as a white circle) from a } \\
\text { central target to } 4 \text { peripheral target locations, in } \\
\text { succession, quickly and accurately (red circles). } \\
\text { Participants hold their position inside the red circle until } \\
\text { a new target appears, which is always at the start } \\
\text { position. A new target will then appear after a brief } \\
\text { hold in the start position and participants then move to } \\
\text { the following target. This task assesses visuomotor } \\
\text { control of the upper limbs. }\end{array}$ \\
\hline & & $\begin{array}{l}\text { Participants control green paddles during this task and } \\
\text { red balls fall towards them from the top of the screen. } \\
\text { Participants are instructed to hit away as many targets } \\
\text { (red circles) as possible, while the task continues to } \\
\text { increase in difficulty (i.e., objects fall more frequently } \\
\text { and with increasing velocity). When a participant } \\
\text { makes contact with a ball, the KINARM robot produces } \\
\text { a force that mimics the force felt by the subject as if } \\
\text { they made contact with an actual object using a real } \\
\text { paddle (unique to this task and OHA below). This task } \\
\text { assesses spatial attention, sensorimotor control and } \\
\text { planning. }\end{array}$ \\
\hline & Object $\mathrm{h}$ & $\begin{array}{l}\text { This task is similar to } \mathrm{OH} \text {, except participants are } \\
\text { instructed to hit only } 2 \text { shapes out of a possible } 8 \text {, while } \\
\text { avoiding distractor targets. Participants are required to } \\
\text { inhibit movements towards distractor targets and only } \\
\text { permit movements to the selected targets. Distractors } \\
\text { do no provide force feedback, as described above, only } \\
\text { the } 2 \text { target shapes. This task assesses attention, rapid } \\
\text { sensorimotor control and inhibition. }\end{array}$ \\
\hline & Ball & $\begin{array}{l}\text { Participants hands are represented by a long white bar } \\
\text { connecting both of their hands, with a white ball resting } \\
\text { on top. Four targets are then presented one at a time in } \\
\text { a diamond pattern and participants move to one of the } \\
4 \text { targets, at a time, in a clockwise direction. } \\
\text { Participants are instructed to move quickly and } \\
\text { accurately to as many target locations as possible } \\
\text { within the } 60 \text { second time limit. This task tested the } \\
\text { bimanual motor coordination. }\end{array}$ \\
\hline Sensory & $\begin{array}{l}\text { Arm position matching } \\
\text { (APM) }\end{array}$ & $\begin{array}{l}\text { This proprioceptive task involved the participants' } \\
\text { dominant arm (i.e., right) being moved by the robot to } \\
\text { one of four preprogrammed coordinates in a square } \\
\text { shape. Participants receive no visual feedback of the } \\
\text { arm being controlled by the robot. After the robot } \\
\text { completes moving the participant's arm, participants } \\
\text { have to mirror-match this position using the } \\
\text { contralateral arm (i.e., left). }\end{array}$ \\
\hline Cognitive & $\begin{array}{l}\text { Reverse visual } \\
\text { reaching ( }\end{array}$ & $\begin{array}{l}\text { This task is similar to VGR; however, visual feedback is } \\
\text { inverted. For example, an arm movement in one } \\
\text { direction (right) would cause the visual feedback on } \\
\text { screen to move in the opposite direction (left). This task } \\
\text { required participants to inhibit their automatic } \\
\text { response to reach towards the spatial goal and instead } \\
\text { move in the opposite direction to achieve the visual } \\
\text { goal. }\end{array}$ \\
\hline
\end{tabular}


tasks. Only a small subset of participants $(n=11)$ completed all 6 KINARM tasks. For more detailed demographics per task, see Table 2 .

\section{Data reduction}

The number of KINARM performance parameters, per task, were substantially reduced (range 67-79\%) by grouping related parameters into components. APM, BonB, VGR, RVGR, and $\mathrm{OH}$ reduced from 9 to 14 performance metrics to 3 components, which accounted for $70-79 \%$ of variance. OHA was reduced from 20 performance items to 5 components, while still accounting for $82 \%$ of the variance (Table 3 ).

\section{Principal component analysis across the KINARM battery}

Descriptions of each task and their respective components are provided below as follows: component name (number of KINARM parameters contributing to that component [according to the $\geq .40$ load criteria mentioned previously], percent of variance explained by that component). See Table 4 for component loadings per task, all available items (i.e., metrics that could be converted to $\mathrm{Z}$ scores) were considered for each PCA per task. For scree plots with parallel analysis per task, see Fig. 2.

\section{Arm position matching}

Scree plot and parallel analysis yielded three models (1-, $2-$, and 3-component solutions) that warranted further examination. For the 2- and 3-component solutions, the use of an orthogonal rotation was justified, as the intercomponent correlations ranged from $|-0.18|$ to $|0.16|$. Both the 1- and 2-component solutions included parameters that did not load onto any of the components (several loadings $<|0.40|$ ) or had sizeable cross loadings. Although several parameters had substantial loadings $(\geq|0.40|)$ on at least one component in the 3-component solution, the 3-component solution was the most interpretable (see Table 4 for the component loadings). The 3 components were related to 1) position accuracy (4, $25 \%), 2)$ movement variability (6, 24\%), and 3) contraction/expansion (3, 21\%).

\section{Ball on bar}

Three models (1-, 2-, and 3-component solutions) were further examined based on the findings of the scree plot and parallel analysis. For the 2- and 3-component solutions, we used an oblique rotation because inter-component correlations ranged from $|0.15|$ to |0.46|. Most parameters had substantial loadings $(\geq|0.40|)$ on at least one component in the 3-component solution. Bar length variability and hand path bias did not load substantially onto any of the 3 components. However, the 3-component solution was the most interpretable (see Table 4 for the component loadings). The three components were associated with 1) speed and success (6,37\%), 2) bar angle (4,19\%) and 3) hand-speed maxima which is related to movement smoothness (3,16\%). The contribution of speed and success to task performance suggests that speed is highly influential for high success rates.

\section{Visually guided reaching}

Scree plot and parallel analysis yielded three models (1-, $2-$, and 3-component solutions) for examination. For the 2- and 3-component solutions, we used an orthogonal rotation (varimax) as inter-component correlations ranged from $|0.06|$ to $|0.16|$. Both 1- and 2-component solutions included a number of parameters that did not load onto any of the components (loadings $<|0.40|$ ) or had sizeable cross loadings, but all parameters had substantial loadings $(\geq|0.40|)$ on at least 1 component in the 3 -component solution. The 3-component solution also was the most interpretable (see Table 4 for the component loadings). The components were related to: 1) initial

Table 2 Participant Demographics and Task Characteristics

\begin{tabular}{|c|c|c|c|c|c|c|c|}
\hline Task & $\mathrm{N}$ & $\begin{array}{l}\text { Mean Age } \\
\text { (range) }\end{array}$ & $\begin{array}{l}\text { Male } \\
\text { Gender (\%) }\end{array}$ & $\begin{array}{l}\text { Dominant } \\
\text { Arm }\end{array}$ & Range of Education & $\begin{array}{l}\text { Mean Number } \\
\text { of Trials [SD] }\end{array}$ & $\begin{array}{l}\text { Mean Task Duration } \\
\text { in seconds [SD] }\end{array}$ \\
\hline Arm Position Matching & 184 & $44.43(18-87)$ & $84(46)$ & Right & $\begin{array}{l}\text { High school - Postdoctoral } \\
\text { research** }\end{array}$ & $25.26[0.98]$ & 88.13 [19.41] \\
\hline Ball on Bar & 208 & $42.16(18-87)$ & $84(40)$ & Right & $\begin{array}{l}\text { High school - Postdoctoral } \\
\text { research*** }\end{array}$ & 1 & $62.57[1.60]$ \\
\hline Visually Guided Reaching & 200 & $42.51(18-88)$ & $80(40)$ & Right & $\begin{array}{l}\text { High school - Postdoctoral } \\
\text { research*** }\end{array}$ & $24[0.07]$ & $102.55[6.83]$ \\
\hline Reverse Visually Guided Reaching & 101 & $41.56(19-81)$ & $46(46)$ & Right & $\begin{array}{l}\text { High school - Post-doctoral } \\
\text { research }\end{array}$ & $30[0.10]$ & $180.27[16.71]$ \\
\hline Object Hit & 190 & $46.28(18-87)$ & $86(45)$ & Right & $\begin{array}{l}\text { High school - Postdoctoral } \\
\text { research** }\end{array}$ & 1 & $138.03[0.43]$ \\
\hline Object Hit and Avoid & 170 & $45.56(18-87)$ & $76(45)$ & Right & $\begin{array}{l}\text { High school - Postdoctoral } \\
\text { research* }\end{array}$ & 1 & $138.29[0.51]$ \\
\hline
\end{tabular}

For range of education, High school reflects completion of grade twelve or completion of secondary school equivalent. ${ }^{*}$ indicate the number of participants that were missing data for that column. For example, ${ }^{*}(n=1),{ }^{* *}(n=2),{ }^{* *}(n=3)$ 
Table 3 Proportion of variance explained by each component across KINARM tasks

\begin{tabular}{|c|c|c|c|c|c|c|c|c|}
\hline \multirow[t]{2}{*}{ Task } & \multirow{2}{*}{$\begin{array}{c}\text { Number of } \\
\text { Parameters } \\
\text { Used for PCA }\end{array}$} & \multicolumn{5}{|c|}{$\begin{array}{c}\text { Variance explained per } \\
\text { component }\end{array}$} & \multirow[t]{2}{*}{$\begin{array}{l}\text { Cumulative } \\
\text { variance }\end{array}$} & \multirow{2}{*}{$\begin{array}{c}\text { \% of data } \\
\text { reduced by } \\
\text { PCA }\end{array}$} \\
\hline & & 1 & 2 & 3 & 4 & 5 & & \\
\hline APM & 12 & $25 \%$ & $24 \%$ & $21 \%$ & & & $70 \%$ & $75 \%$ \\
\hline BonB & 14 & $37 \%$ & $19 \%$ & $16 \%$ & & & $72 \%$ & $79 \%$ \\
\hline VGR & 9 & $34 \%$ & $30 \%$ & $15 \%$ & & & $79 \%$ & $67 \%$ \\
\hline RVGR & 11 & $38 \%$ & $24 \%$ & $12 \%$ & & & $74 \%$ & $73 \%$ \\
\hline $\mathrm{OH}$ & 14 & $27 \%$ & $26 \%$ & $18 \%$ & & & $71 \%$ & $79 \%$ \\
\hline OHA & 20 & $21 \%$ & $20 \%$ & $18 \%$ & $16 \%$ & $7 \%$ & $82 \%$ & $75 \%$ \\
\hline
\end{tabular}

Grey shaded region indicates that there are no components for these tasks. APM Arm position matching, BonB Ball on bar, VGR Visually guided reaching, RVGR Reverse visually guided reaching, $\mathrm{OH}$ Object hit, $\mathrm{OHA}$ Object hit and avoid

movement and smoothness (5, 34\%), 2) total movement (5, $30 \%)$, and 3 ) posture and reaction time (3, 15\%).

\section{Reverse visually guided reaching}

Scree plot and parallel analysis yielded three models (1-, $2-$, and 3-component solutions) for further examination. For the 2- and 3-component solutions, we used an orthogonal rotation, as the inter-component correlations ranged from $|-0.09|$ to $|0.13|$. Both the 1 - and 2-component solutions included items that did not load onto any of the components (all loadings $<|0.40|$ ), but all items had substantial loadings $(\geq|0.40|)$ on at least one component in the 3-component solution. The 3-component solution also was the most interpretable (see Table 4 for the component loadings). The 3 components were associated with: 1) initial movement and smoothness (7, 38\%), 2) total movement $(5,24 \%)$, and 3 ) reaction time (1, 12\%). Initial movement and smoothness accounted for more variance in this task than it did in VGR. Therefore, initial movement is imperative for success in RVGR.

\section{Object hit}

According to the scree plot and parallel analysis, three models (1-, 2-, and 3-component solutions) warranted further examination. For the 2- and 3-component solutions, we used an orthogonal rotation, as the inter-component correlations ranged from $|0.03|$ to $|0.24|$. Both the 1- and 2-component solutions included items that did not load onto any of the components (loadings $<|0.40|$ ) or had sizeable cross loadings, but all items had substantial loadings $(\geq|0.40|)$ on at least one component in the 3-component solution. The 3-component solution also was the most interpretable (see Table 4 for the component loadings). The 3 components were comprised of: 1) hand bias (6, 27\%), 2) speed and area (5, 26\%), and 3) accuracy (4, 18\%). Hits with left and right hands cross loaded onto both accuracy and hand bias.

\section{Object hit and avoid}

According to the scree plot and parallel analysis, five models (1-, 2-, 3-, 4- and 5-component solutions) warranted further examination. For the 2-, 3-, 4-, and 5-component solutions, we kept the oblique rotation because the inter-component correlations ranged from $|-0.12|$ to $|0.30|$. The 1-, 2-, 3-, and 4-component solutions included items that did not load onto any of the components (all loadings $<|0.40|$ ) or had substantial cross loadings, but all items had substantial loadings (> $|0.40|)$ on at least one component in the 5-component solution, which was also the most interpretable (see Table 4 for the component loadings). The 5 components were related to: 1) impulsivity (6, 21\%), 2) accuracy (6, $20 \%), 3)$ hand bias $(6,18 \%), 4)$ speed and area (5, 16\%), and 5) miss bias (1, 7\%). Three of these components (hand bias, speed and area, and accuracy) are shared with $\mathrm{OH}$, whereas miss bias and distractor avoidance are components unique to OHA.

\section{Discussion}

The KINARM robot produces granular datasets of performance metrics associated with sensory, motor, visuospatial, and executive function. The primary objective of this study was to reduce multivariate data produced by the KINARM and generate interpretable and intuitively understandable components across 6 behavioral tasks to improve characterization of participant performance. KINARM data dimensionality was substantially reduced, while still retaining a large proportion of variance. Therefore, complex participant data produced by the KINARM robot can be reduced into a small number of components that characterize participant performance.

\section{Interpretability and classification of components}

We initially used PCA to reduce our multivariate dataset, and we then investigated if this statistical technique identified interpretable and classifiable components of participant performance. Within a task, components explained intuitive performance metrics. For example, in APM (a task that assesses limb proprioception), the 3 components identified were distinct measures of function (position accuracy, movement variability and contraction/expansion). Furthermore, KINARM parameters were separated reproducibly across multiple tasks. For 
Table 4 Principal component loadings for KINARM behavioral battery

\begin{tabular}{|c|c|c|c|c|c|c|}
\hline \multirow[t]{2}{*}{ Task } & \multirow[t]{2}{*}{ KINARM Items } & \multicolumn{5}{|c|}{ Component Loadings } \\
\hline & & 1 & 2 & 3 & 4 & 5 \\
\hline \multirow[t]{12}{*}{ Arm Position Matching } & Absolute Error $X$ & 0.870 & 0.210 & -0.040 & & \\
\hline & Absolute Error Y & 0.320 & 0.400 & -0.230 & & \\
\hline & Absolute Error XY & 0.910 & 0.250 & -0.100 & & \\
\hline & Variability $X$ & 0.260 & 0.810 & -0.130 & & \\
\hline & Variability Y & 0.140 & 0.750 & -0.060 & & \\
\hline & Variability XY & 0.270 & 0.860 & -0.140 & & \\
\hline & Contraction Expansion Ratio X & -0.120 & -0.040 & 0.890 & & \\
\hline & Contraction Expansion Ratio Y & 0.020 & -0.140 & 0.800 & & \\
\hline & Contraction Expansion Ratio XY & -0.060 & -0.050 & 0.990 & & \\
\hline & Shift $X$ & -0.550 & 0.580 & -0.070 & & \\
\hline & Shift $Y$ & 0.250 & -0.580 & -0.070 & & \\
\hline & Shift XY & 0.870 & -0.030 & -0.040 & & \\
\hline \multirow[t]{14}{*}{ Ball on Bar } & Targets completed & 0.997 & -0.146 & 0.001 & & \\
\hline & Mean movement time & -0.965 & 0.066 & -0.037 & & \\
\hline & Mean ball speed & 0.942 & 0.083 & 0.029 & & \\
\hline & Mean right hand speed & 0.913 & 0.164 & -0.017 & & \\
\hline & Mean left hand speed & 0.956 & 0.045 & 0.020 & & \\
\hline & Right hand speed maxima & 0.054 & -0.012 & 0.950 & & \\
\hline & Left hand speed maxima & 0.175 & -0.069 & 0.766 & & \\
\hline & Mean bar angle rad & -0.067 & 0.633 & -0.045 & & \\
\hline & Stdev bar angle rad & -0.056 & 0.801 & -0.201 & & \\
\hline & Bar length variability* & 0.250 & 0.335 & 0.145 & & \\
\hline & Hand speed diff & 0.473 & 0.639 & 0.108 & & \\
\hline & Norm absolute hand speed diff & 0.027 & 0.849 & 0.166 & & \\
\hline & Hand speed peak bias & -0.263 & 0.113 & 0.732 & & \\
\hline & Hand path bias* & -0.098 & 0.342 & -0.191 & & \\
\hline \multirow[t]{9}{*}{ Visually Guided Reaching } & Posture speed & -0.077 & 0.519 & 0.512 & & \\
\hline & Reaction time & 0.078 & -0.162 & 0.863 & & \\
\hline & Initial direction error & 0.547 & 0.291 & 0.435 & & \\
\hline & Initial distance ratio & -0.915 & 0.073 & -0.096 & & \\
\hline & Speed maxima count & 0.780 & -0.324 & -0.323 & & \\
\hline & Min max speed difference & 0.738 & 0.568 & 0.098 & & \\
\hline & Movement time & 0.105 & -0.950 & -0.035 & & \\
\hline & Path length ratio & 0.773 & 0.485 & 0.142 & & \\
\hline & Max speed & 0.371 & 0.847 & -0.148 & & \\
\hline \multirow[t]{8}{*}{ Reverse Visually Guided Reaching } & Posture speed & -0.050 & 0.614 & 0.394 & & \\
\hline & Reaction time & 0.022 & -0.016 & 0.800 & & \\
\hline & Initial direction error & 0.818 & 0.224 & 0.055 & & \\
\hline & Initial distance ratio & -0.856 & 0.133 & -0.343 & & \\
\hline & Initial speed ratio & -0.806 & -0.023 & 0.316 & & \\
\hline & Speed maxima count & 0.837 & -0.101 & 0.302 & & \\
\hline & Min max speed difference & 0.263 & 0.721 & 0.053 & & \\
\hline & Movement time & 0.621 & -0.680 & 0.229 & & \\
\hline
\end{tabular}


Table 4 Principal component loadings for KINARM behavioral battery (Continued)

\begin{tabular}{|c|c|c|c|c|c|c|}
\hline \multirow[t]{2}{*}{ Task } & \multirow[t]{2}{*}{ KINARM Items } & \multicolumn{5}{|c|}{ Component Loadings } \\
\hline & & 1 & 2 & 3 & 4 & 5 \\
\hline & Path length ratio & 0.547 & 0.692 & -0.013 & & \\
\hline & Max speed & -0.248 & 0.871 & -0.303 & & \\
\hline & Correction time & 0.788 & 0.095 & -0.330 & & \\
\hline \multirow[t]{14}{*}{ Object Hit } & Total hits & -0.028 & 0.137 & 0.932 & & \\
\hline & Hits with left & -0.612 & 0.053 & 0.658 & & \\
\hline & Hits with right & 0.545 & 0.218 & 0.758 & & \\
\hline & Median error & -0.046 & 0.119 & 0.647 & & \\
\hline & Miss bias ${ }^{a}$ & -0.040 & -0.022 & -0.365 & & \\
\hline & Right hand speed & 0.322 & 0.847 & 0.204 & & \\
\hline & Movement area (right) & 0.200 & 0.881 & 0.052 & & \\
\hline & Left hand speed & -0.148 & 0.888 & 0.132 & & \\
\hline & Movement area (left) & -0.219 & 0.915 & 0.061 & & \\
\hline & Hand bias of hits & 0.893 & 0.166 & 0.082 & & \\
\hline & Hand transition & -0.863 & -0.008 & 0.051 & & \\
\hline & Hand selection overlap & -0.056 & 0.612 & 0.094 & & \\
\hline & Hand speed bias & 0.876 & 0.037 & 0.140 & & \\
\hline & Movement area bias & 0.719 & -0.290 & -0.063 & & \\
\hline \multirow[t]{20}{*}{ Object Hit and Avoid } & Total hits & -0.125 & 0.933 & 0.037 & 0.067 & 0.006 \\
\hline & Hits with left & -0.092 & 0.780 & -0.453 & 0.034 & 0.233 \\
\hline & Hits with right & -0.061 & 0.748 & 0.483 & 0.070 & -0.210 \\
\hline & Total Distractor hits & 0.982 & 0.009 & 0.013 & 0.036 & -0.012 \\
\hline & Distractor hits (left) & 0.925 & 0.034 & -0.020 & -0.025 & 0.019 \\
\hline & Distractor hits (right) & 0.896 & -0.001 & 0.033 & 0.070 & -0.027 \\
\hline & Median error & -0.414 & 0.419 & 0.076 & 0.073 & -0.012 \\
\hline & Miss bias & -0.043 & 0.034 & 0.063 & 0.008 & 0.914 \\
\hline & Right hand speed & 0.133 & 0.239 & 0.304 & 0.724 & -0.025 \\
\hline & Movement area (right) & 0.004 & -0.054 & 0.360 & 0.854 & 0.050 \\
\hline & Left hand speed & 0.133 & 0.215 & -0.241 & 0.764 & 0.136 \\
\hline & Movement area (left) & -0.010 & -0.035 & -0.327 & 0.922 & -0.125 \\
\hline & Hand bias of hits & -0.010 & 0.042 & 0.809 & 0.058 & -0.387 \\
\hline & Hand transition & -0.060 & 0.030 & -0.824 & -0.082 & -0.390 \\
\hline & Hand selection overlap & 0.021 & -0.291 & 0.107 & 0.568 & 0.124 \\
\hline & Hand speed bias & 0.003 & 0.045 & 0.840 & 0.007 & -0.238 \\
\hline & Movement area bias & 0.029 & -0.029 & 0.860 & -0.131 & 0.239 \\
\hline & Objects hit & 0.484 & 0.856 & 0.003 & 0.037 & -0.041 \\
\hline & Distractor proportion & 0.963 & -0.112 & -0.003 & 0.027 & -0.005 \\
\hline & Object processing rate & -0.380 & 0.799 & 0.000 & 0.071 & 0.041 \\
\hline
\end{tabular}

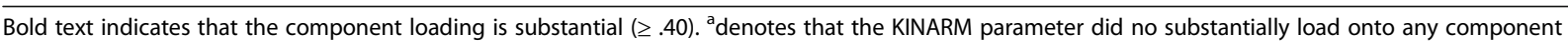

example, OHA was divided into 5 components: impulsivity, accuracy, hand bias, speed and area, and miss bias. Three of these components (accuracy, hand bias, and speed and area) are comparable to the PCA results observed from the $\mathrm{OH}$ task, thereby providing evidence that these tasks assess similar underlying behaviors.
However, despite assessing similar underlying behaviors, the variance explained by each component differs. For example, speed and area accounts for more variability than accuracy, whereas in OHA accuracy accounts for more variability than speed and area. The addition of two unique components (i.e., miss bias and impulsivity) 
a

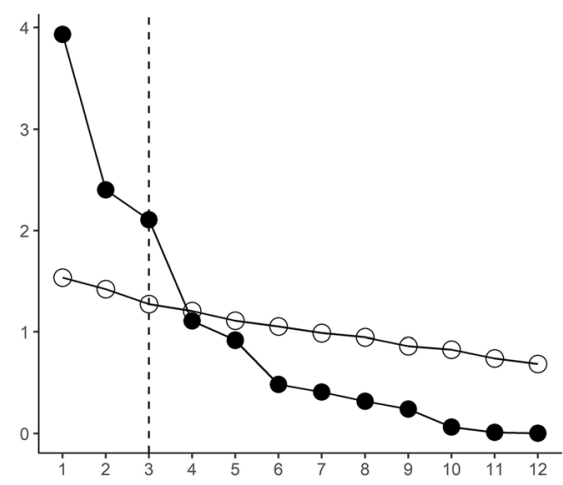

C

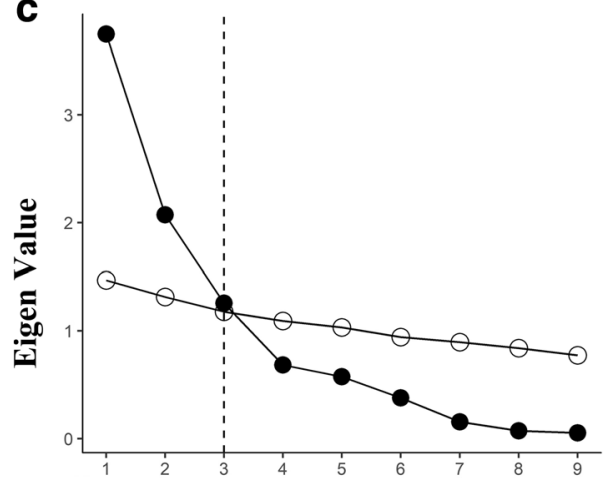

e

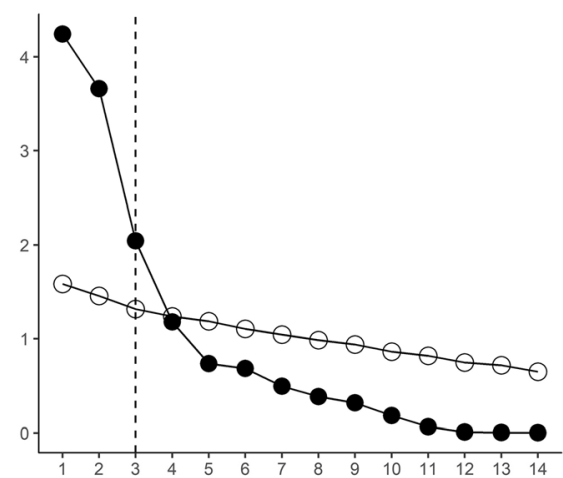

b

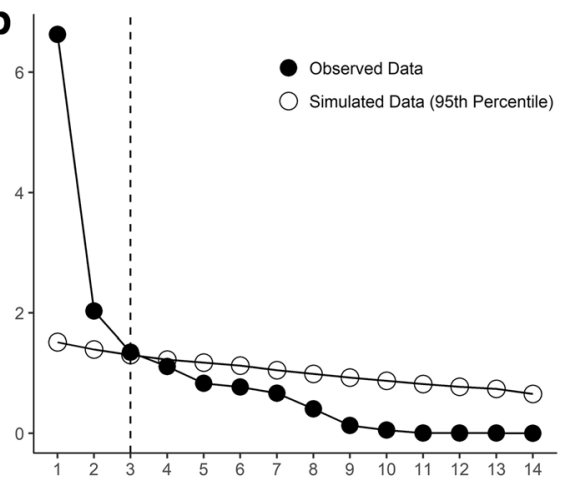

d

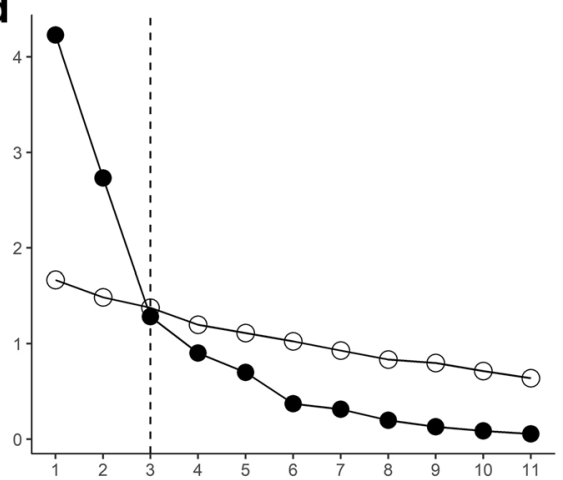

f

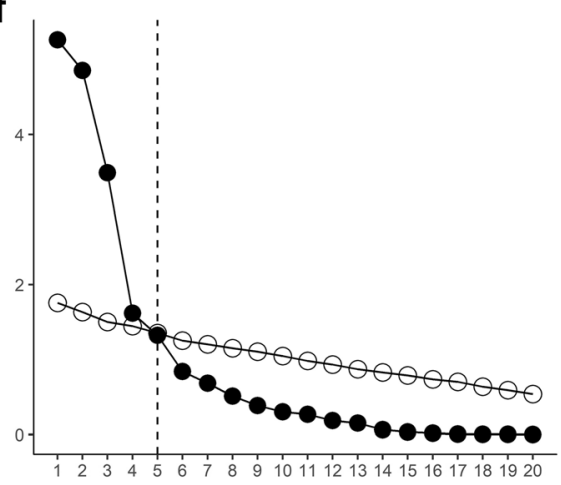

\section{Component Number}

Fig. 2 Scree plots with parallel analysis indicating the number of components to be selected across KINARM tasks. a Arm Position Matching; b Ball on Bar; c Visually Guided Reaching; d Reverse Visually Guided Reaching; e Object Hit; f Object Hit and Avoid

during the OHA may quantitatively represent the increase in cognitive load during this task. These two components that are absent from $\mathrm{OH}$, and which account for $28 \%$ of overall variability, likely reflect the different impacts of impulsive movements in $\mathrm{OH}$ and $\mathrm{OHA}$. In $\mathrm{OH}$, quick and effectively impulsive movements are rewarded; quick movements with little inhibition will result in many targets being hit. In contrast, in OHA there is a penalty for impulsive movements that are not processed thoroughly prior to execution (i.e., deciding whether or not an object is a target or distractor prior to executing a movement towards it). Furthermore, behaviorally during the $\mathrm{OH}$ task, the subject can hit a total of
300 targets during the trial. In contrast, there are only 200 targets during OHA, plus 100 distractors. Therefore, hand bias and speed and area account for less variability in OHA, as now the subjects must inhibit their automatic responses (i.e., impulsivity), which also results in an increased need for improved accuracy. This contrast in cognitive load between tasks may anecdotally account for the difference in variance explained by the components mentioned above across $\mathrm{OH}$ and $\mathrm{OHA}$. This result of similar inter-task grouping of parameters across related KINARM tasks was also observed during VGR and RVGR. Taken together, our results demonstrate the potential utility of PCA when applied to granular datasets, 
as well as the increased interpretability of KINARM parameters when categorized into behaviorally meaningful components that characterize performance.

Furthermore, parameter associations, positive or negative, strong or weak, within their respective components were logically related to participant performance. For example, the PCA of the BonB task identified the component speed and success. Targets completed, mean ball speed, mean right- and left-hand speed, and hand speed difference all had substantial positive loadings, whereas mean movement time had a substantial negative loading. Therefore, as participants increased their right- and left-hand speed, and thus ball speed, participants decrease the time from a target being displayed on screen to the target being reached by the participant, which results in a greater number of targets being completed throughout the task. In addition, the parameter norm absolute hand speed difference loaded substantially on bar angle but had a very low loading on speed and area. Behaviorally, this result is intuitive. If a participant reacts to a target quickly, the bar angle may become skewed if not carefully balanced, which is reflected by the substantial positive loadings of the other angle related parameters onto this component. These direction-based associations, low or high, were observed across most KINARM tasks and their respective parameters. These components will need to be further validated across KINARM platforms and patient populations. Overall, our analysis produced a consistent inter-task classification of behavioural variables (e.g., VGR and RVGR, OH and $\mathrm{OHA}$ ), which demonstrates that these tasks assess similar underlying behaviours and further supports the validity of using this statistical technique on KINARM performance metrics. Our current analysis has substantially reduced these granular datasets into biologically plausible, interpretable, and behaviorally meaningful components.

\section{Cross-loading of parameters with multiple components}

PCA indicated that some of the KINARM parameters cross-loaded with multiple components. For example, the APM task identified 3 components (position accuracy, movement variability, and contraction/expansion), which is fairly consistent with previous research that described these 3 variables as observed patterns of impairment after stroke [2]. Despite mostly a strong separation of these three components, shift $\mathrm{x}$ loaded onto both the position accuracy and movement variability components, with an inverse sign from negative to positive respectively. Shift indicates a systematic bias to move either left/right or front/back in the workspace. Therefore, in the position accuracy component, shift X (left/right) indicates, with a negative association, that shift $\mathrm{X}$ biases to the left as absolute error in the $\mathrm{X}$ and
$\mathrm{XY}$ plane increases. In contrast, component 2 , movement variability, indicates that as $\mathrm{X}$ shifts in the positive direction (i.e., hand moves to the right), overall variability increases.

Furthermore, certain parameters did not intuitively cross-load onto multiple components. For example, the $\mathrm{OH}$ task identified 3 components (hand bias, speed and area, and accuracy). Unexpectedly, the parameter total hits, which should be intuitively related to hits with left and right, did not cross-load onto the accuracy and hand bias components. However, this result demonstrates that total hits is highly related to accuracy, which is separable from hand bias. These components are not highly interrelated, as indicated by the use of orthogonal rotation, and measure separable aspects of participant performance. Therefore, hand bias measures the bias towards the participants limb preference, and use in space, which is separable from overall accuracy and the total targets being hit (e.g., right-handed participants may have biased use of their right hand, but this is separable from the number of targets that were accurately hit using either hand).

Interestingly, only three parameters did not substantially load onto any component: miss bias of $\mathrm{OH}$ and bar length variability and hand path bias from the BonB task. The reason for these low loadings is unclear. However, miss bias loaded highly onto a separate component for the OHA task, which may indicate that any bias of misses toward one side of the work space, or the other, is related to the increase in cognitive load during OHA. Furthermore, the low loadings for bar length variability and hand path bias may be the consequence of only analyzing level one of BonB, as this task increases with difficulty in the subsequent levels [19]. However, previous research has identified that level one of BonB identified the most performance impairments (i.e., highest number of parameters failed) in stroke participants, relative to control subjects [19]. Furthermore, the number of parameters failed tended to decrease with increasing task difficulty in successive levels, potentially due to the increased variability observed among controls, which ultimately influenced cut-off criteria used to quantify impairment among stroke participants [19]. Therefore, level one of BonB may be the most sensitive level used to detect impairment and is the most relevant for the current analysis. Future analysis may need to apply PCA to each level separately to investigate if the components are similar across all three levels. However, despite identifying some parameters that cross-load with multiple components and only a couple that did not substantially load onto any component, the majority of KINARM performance metrics did not cross-load with one another. Most components demonstrated high loadings with their respective components, which indicates a strong 
separation of components that quantify participant performance.

\section{Limitations and future directions}

We excluded left-hand dominant participants, as many participants were right-handed, which means our findings may not generalize to left-handed participants. Furthermore, we could not conduct PCA across all task parameters, as participants did not complete all KINARM tasks. Therefore, we were unable to conduct PCA on broadly pooled performance scores across tasks, which confined our results to descriptions of each task only. However, the PCA has yielded task specific separations of components to further characterize sensorimotor function among healthy subjects, which may serve as a normative data set for future clinical comparisons of subject performance. In addition, two to three participants per task, except for RVGR, were missing participant data for education obtained. However, these missing data should not substantially impair our range of education. In addition, some tasks do not have Z-scores for all parameters being recorded (these metrics could not be standardized), and thus these parameters were excluded from this analysis. We conducted PCA only on the End-Point robot data, and therefore, we will need to conduct PCA on healthy participant data generated using the KINARM Exoskeleton. It is unclear how antigravity support provided by the Exoskeleton will affect participant performance and the generated components. Therefore, PCA of Exoskeleton data will be imperative to future investigations to implement PCA across KINARM platforms. Also, the current analysis was conducted in healthy participants, and future PCA will need to examine participant performance using a clinical sample, such as stroke, to validate these components and characterize performance in clinical populations to further demonstrate the utility of our current findings. Furthermore, the current analysis does not address individual or group differences in our healthy participant sample, which may complicate future comparisons between healthy subjects and clinical populations. However, using PCA across 6 KINARM tasks, which assesses a broad range of upper limb sensorimotor function, has led to a substantial reduction in the dimensionality of our data, and produced interpretable components of performance.

Therefore, PCA of KINARM data has the potential to become a valuable clinical tool. Applying PCA and identifying main sources of variability in a clinical examination can help make data from research tools, such as the KINARM, more concise and easily interpretable. Furthermore, identifying sources of strong variability may improve the detection of fluctuations in performance, which could increase the clinical relevance of robotic assessment as an evaluation tool. Therefore, analyzing data produced by a clinical population offers the potential to increase the clinical utility of the KINARM robot by maximizing interpretability of participant performance, while also minimizing information loss, to increase the characterization of performance among various populations. It is not clear if patient performance will result in similar components of performance patterns as healthy participants. Therefore, future applications of this analysis may offer potential insight into specific patterns of sensorimotor impairment among patient populations.

\section{Conclusions}

Using PCA, granular KINARM performance data can be substantially reduced to a small number of interpretable components with minimal information loss and still accounting for a large amount of variance. To validate our current findings and to further characterize sensorimotor function and impairment, the components derived from our sample of healthy participants will serve as normative data for future comparisons to patient populations.

\section{Additional files}

Additional file 1: Volunteer screening checklist. This form was administered to participants by trained researchers to screen for previous neurological and musculoskeletal complications. (PDF 53 kb)

Additional file 2: KINARM Standard Tests Parameter Tables. These tables provide detailed descriptions of each parameter per KINARM task. These tables were extracted from Dexterit-E 3.6 User Guide with permission from BKIN Technologies Ltd. (PDF 145 kb)

Additional file 3: Correlation matrices of KINARM parameters across all tasks. (DOCX $30 \mathrm{~kb}$ )

\section{Abbreviations}

APM: Arm position matching; BonB: Ball on bar; KINARM: Kinesiological instrument for normal and altered reaching movements; $\mathrm{OH}$ : Object Hit; OH\&A: Object hit and avoid; PCA: Principal component analysis;

RVGR: Reverse visually guided reaching; VGR: Visually guided reaching

\section{Acknowledgments}

The authors would like to thank Kim Moore and Simone Appaqaq for assessing the control participants, as well as Helen Bretzke and Justin Peterson for their contribution to software implementation and database management.

\section{Funding}

Funding provided by an Ontario Research Foundation - Research Excellence grant and a GSK-CIHR Chair in Neuroscience to SHS. The funding agencies had no role in the design of this study and they did not have any role in data collection or analysis.

\section{Availability of data and materials}

The datasets used and/or analysed during the current study are available from the corresponding author on reasonable request.

\section{Authors' contributions}

MDW participated in the statistical plan, data analysis and drafting of the manuscript. LERS participated in data analysis and drafting of the manuscript. SHS participated in study design and drafting of the manuscript. JGB participated in study design and drafting of the manuscript. JAJ is the primary investigator. She participated in the statistical plan, reproducing the 
data analysis and results, and drafting of the manuscript. All authors read and approved the final manuscript.

\section{Ethics approval and consent to participate}

The Queen's University and Affiliated Hospitals Health Sciences Research Ethics Board approved recruitment and assessment of these participants. Informed consent was obtained from each participant prior to the KINARM assessment.

\section{Consent for publication}

Not applicable.

\section{Competing interests}

MDW has nothing to disclose.

LERS has nothing to disclose.

SHS is the cofounder of BKIN Technologies, the manufacturer of the KINARM device.

JGB receives a stipend from the Trillium Gift of Life Network to support his role as the Hospital Donation Support Physician.

JAJ has nothing to disclose.

\section{Publisher's Note}

Springer Nature remains neutral with regard to jurisdictional claims in published maps and institutional affiliations.

\section{Author details}

${ }^{1}$ Centre for Neuroscience Studies, Queen's University, Botterell Hall, 18 Stuart St, Kingston, ON, Canada. 'Department of Critical Care Medicine, Queen's University, Kingston, ON, Canada. ${ }^{3}$ Department of Medicine, Queen's University, Kingston, ON, Canada. ${ }^{4}$ Department of Psychology, Queen's University, 318 Craine Hall, 62 Arch Street, Kingston, ON K7L 3N6, Canada.

Received: 17 October 2017 Accepted: 13 July 2018

Published online: 31 July 2018

\section{References}

1. Coderre AM, et al. Assessment of upper-limb sensorimotor function of subacute stroke patients using visually guided reaching. Neurorehabil Neural Repair. 2010;24:528-41.

2. Dukelow SP, et al. Quantitative assessment of limb position sense following stroke. Neurorehabil Neural Repair. 2010;24:178-87.

3. Dukelow SP, Herter TM, Bagg SD, Scott SH. The independence of deficits in position sense and visually guided reaching following stroke. J Neuroeng Rehabil. 2012;9:72.

4. Debert CT, Herter TM, Scott SH, Dukelow S. Robotic assessment of sensorimotor deficits after traumatic brain injury. J Neurol Phys Ther. 2012; 36:58-67.

5. Williams $L$, et al. Sensory-motor deficits in children with fetal alcohol Spectrum disorder assessed using a robotic virtual reality platform. Alcohol Clin Exp Res. 2014;38:116-25.

6. Pearson K. On Lines and Planes of Closest Fit to Systems of Points in Space. Philos Mag Ser 6. 1901;2:559-72.

7. Hotelling $\mathrm{H}$. Analysis of a complex of statistical variables into principal components. J Educ Psychol. 1933;24:417-41.

8. Ringner M. What is principal component analysis? Nat Biotechnol. 2008;36: 303-4.

9. Joliffe IT, Cadima J. Principal component analysis: a review and recent developments. Phil Trans R Soc A. 2016;374:20150202.

10. BKIN Technologies Ltd, Kingston, ON, Canada. Dexterit-E User Guide v 3.6 KINARM Standard Tests Parameter Definitions. http://www.bkintechnologies. com/download/dexterit-e-user-guide-v-3-6-kinarm-standard-tests-parameterdefinitions/. Accessed 2 Oct 2017

11. Team RC. R: a language and environment for statistical computing. Vienna, Austria: R Foundation for Statistical Computing; 2016. https//www.R-project.org/

12. Revelle, W. Psych: Procedures for Personality and Psychol Res. Evanston: Northwestern University; 2017. https://cran.r-project.org/package=psych.

13. Sakaluk JK, Short SD. A methodological review of exploratory factors analysis in sexuality research: used practices, best practices, and data analysis resources. I Sex Res. 2017;54:1-9.

14. Cattell RB. The scree test for the number of factors. Multivariate Behav Res. 1966;1:245-76.
15. O'connor BP. SPSS and SAS programs for determining the number of components using parallel analysis and Velicer's MAP test. Behav Res Methods Instrum Comput. 2000;32:396-402.

16. Horn JL. A rationale and test for the number of factors in factor analysis. Psychometrika. 1965;30:179-85.

17. Jennrich RI, Sampson PF. Rotation for simple loadings. Psychometrika. 1966; 31:313-23.

18. Kaiser HF. The varimax criterion for analytic rotation in factor analysis. Psychometrika. 1958;23:187-200.

19. Lowrey CR, Jackson CPT, Bagg S, Dukelow SP, Scott SH. A novel robotic task for assessing impairments in bimanual coordination post-stroke. Int J Phys Med Rehabil. 2014;S3:002.

\section{Ready to submit your research? Choose BMC and benefit from:}

- fast, convenient online submission

- thorough peer review by experienced researchers in your field

- rapid publication on acceptance

- support for research data, including large and complex data types

- gold Open Access which fosters wider collaboration and increased citations

- maximum visibility for your research: over $100 \mathrm{M}$ website views per year

At BMC, research is always in progress.

Learn more biomedcentral.com/submissions 